\title{
DISEÑO DE UN SISTEMA DE EVALUACIÓN FORMATIVA EN EL ÁREA DE LENGUA CASTELLANA Y LITERATURA
}

Design of a formative evaluation system in the area of Spanish Language and Literature

Desenho de um sistema de avaliação formativa na área de Língua Castelhana e

Literatura

\section{Rosa Ortiz de Santos (1)}

Noelia Santamaría-Cárdaba (2)

(1) Universidad de Valladolid, España. Correo electrónico: rosa.ortiz@uva.es

(2) Universidad de Valladolid, España. Correo electrónico: noelia.santamaria.cardaba@uva.es

\section{Resumen}

La evaluación formativa y compartida es un eficaz medio para la mejora de los procesos de enseñanza y aprendizaje en todos los niveles educativos. Partiendo de esta premisa, se convierte en tarea irremplazable el diseño de sistemas de evaluación adecuados a las características de cada realidad educativa. En esta aportación diseñamos y presentamos un sistema de evaluación formativa para el área de Lengua Castellana y Literatura del tercer curso de Educación Primaria. En el sistema se incluyen instrumentos interrelacionados, orientados a la autoevaluación, a la heteroevaluación y a la coevaluación, que favorecen la implicación y participación del estudiantado en su propio proceso de aprendizaje y mejora la relación entre los diferentes agentes educativos. La propuesta se ha diseñado en base a un contexto hipotético, lo que imposibilita evaluar su eficacia. No obstante, consideramos que podría ser un modelo de evaluación, transferible a cualquier nivel educativo y/o área disciplinar de Educación Primaria.

Palabras clave: Evaluación formativa; lengua castellana y literatura; Educación Primaria; instrumento de evaluación; calificación

\begin{abstract}
Formative and shared assessment is an effective means of improving teaching and learning processes at all levels of education. Starting from this premise, the design of evaluation systems adapted to the characteristics of each educational reality becomes an
\end{abstract}


irreplaceable task. In this contribution we designed and presented a formative evaluation system for the area of Spanish Language and Literature in the third year of Primary Education. The system includes interrelated instruments, aimed at self-evaluation, heteroevaluation and co-evaluation, which favour the involvement and participation of students in their own learning process and improve the relationship between the different educational agents. The proposal has been designed based on a hypothetical context, which makes it impossible to evaluate its effectiveness. Nevertheless, we consider that it could be an evaluation model, transferable to any educational level and/or disciplinary area of Primary Education.

Keywords: Formative assessment; spanish language and literature; Primary Education; assessment instrument; grading

\section{Resumo}

A avaliação formativa e partilhada é um meio eficaz para melhorar os processos de ensino e aprendizagem em todos os níveis de ensino. Partindo desta premissa, o desenho de sistemas de avaliação adaptados às características de cada realidade educativa tornase uma tarefa insubstituível. Nesta contribuição, desenhamos e apresentamos um sistema de avaliação formativa na área de Língua Castelhana e Literatura no terceiro ano do Ensino Primário. O sistema inclui instrumentos interrelacionados, orientados à autoavaliação, heteroavaliação e coavaliação, que favorecem o envolvimento e a participação dos alunos no seu próprio processo de aprendizagem e melhoram a relação entre os diferentes agentes educativos. A proposta foi concebida com base num contexto hipotético, o que torna impossível avaliar a sua eficácia. Não obstante, consideramos que poderia ser um modelo de avaliação transferível a qualquer nível de ensino e/ou área disciplinar do Ensino Primário.

Palavras-chave: Avaliação formativa; língua castelhana e literatura; Educação Primária; instrumento de avaliação; classificação

\section{Introducción}

La finalidad de la evaluación formativa es "mejorar los procesos de E-A ${ }^{1}[\ldots]$; está orientada a que el alumnado aprenda más y a que el profesorado aprenda a mejorar su

\footnotetext{
${ }^{1}$ Enseñanza-Aprendizaje (E-A)
} 
práctica docente" (López-Pastor, 2012, p. 120). En este sentido, la evaluación es "el arma más poderosa [...] para influir en el modo en el que los estudiantes responden a las asignaturas y se comportan como alumnos" (citado en Hamodi, López-Pastor, \& LópezPastor, 2015, p. 72).

Los beneficios de emplear un modelo de evaluación formativa son cuantiosos; favorece la adecuación al aprendizaje del alumnado y mejora el rendimiento y el aprendizaje (Gargallo, Suárez, \& Ferreras, 2007; Fraile, López-Pastor, Castejón, \& Romero, 2013). Para ello, existe la necesidad de diseñar instrumentos que faciliten este tipo de evaluación (López-Pastor \& Pérez-Pueyo, 2017). Esta es la relevancia de la propuesta, en la que se diseña y presenta un sistema de evaluación.

\section{Contextualización}

La propuesta de evaluación formativa está dirigida al primer trimestre de Lengua Castellana y Literatura, del tercer curso de Educación Primaria.

\section{Diseño y desarrollo}

Las técnicas e instrumentos del sistema de evaluación se muestran en la Tabla 1.

Tabla 1.

Técnicas e instrumentos de evaluación

\begin{tabular}{|c|c|c|c|c|}
\hline & Técnica & Instrumento & Cantidad & Tiempo \\
\hline \multirow{6}{*}{$\begin{array}{l}\text { Tareas del } \\
\text { alumnado }\end{array}$} & Autoevaluación & Informe de talleres & 2 por UD & $15 \mathrm{~min}$ \\
\hline & Coevaluación & Asamblea de talleres & 2 por UD & $15 \mathrm{~min}$ \\
\hline & Autoevaluación & Diana de autoevaluación & 1 por UD & $5 \mathrm{~min}$ \\
\hline & Autoevaluación & Autocorrección del ejercicio escrito & 1 por UD & $15-20 \mathrm{~min}$ \\
\hline & Autoevaluación & Ficha de autoevaluación del dossier & 1 por trimestre & $15 \mathrm{~min}$ \\
\hline & Heteroevaluación & $\begin{array}{l}\text { Cuestionario de evaluación: práctica } \\
\text { docente y proceso de E-A. }\end{array}$ & 1 por trimestre & $10 \mathrm{~min}$ \\
\hline \multirow[t]{5}{*}{$\begin{array}{l}\text { Tareas del } \\
\text { docente }\end{array}$} & $\begin{array}{l}\text { Autoevaluación - } \\
\text { heteroevaluación }\end{array}$ & Cuaderno del profesor & Trimestre & Imprevisible \\
\hline & Observación & Registro anecdótico & & \\
\hline & Heteroevaluación & Ejercicios escritos & 1 por UD & Imprevisible \\
\hline & Heteroevaluación & Dossier del alumnado & Trimestre & Imprevisible \\
\hline & Autoevaluación & $\begin{array}{l}\text { Ficha de autoevaluación trimestral y } \\
\text { de evaluación de la enseñanza }\end{array}$ & 1 por trimestre & $10 \mathrm{~min}$ \\
\hline
\end{tabular}

Respecto a los instrumentos, cada estudiante tendrá una carpeta de trabajo con las producciones de la asignatura, una copia de los informes de los talleres, los ejercicios escritos, y una ficha de autoevaluación trimestral (Figura 1). Los ejercicios escritos individuales sirven para evaluar habilidades de comprensión lectora, expresión escrita y la adquisición de contenidos teóricos. Los discentes podrán utilizar el material de sus 
carpetas y la corrección no incluirá calificación; consistirá en propuestas de mejora para que el alumnado se autocorrija la tarea. Tras la reelaboración (20 minutos, sin material de apoyo), el docente realizará la corrección final con nuevas anotaciones y reflejará en su ficha de criterios de evaluación su grado de consecución. Al final se incluirá una diana de autoevaluación (Figura 2) para que el discente valore su aprendizaje; los indicadores de valoración coinciden con los objetivos de aprendizaje y los criterios de evaluación establecidos por la legislación para el área y nivel educativo abordado.

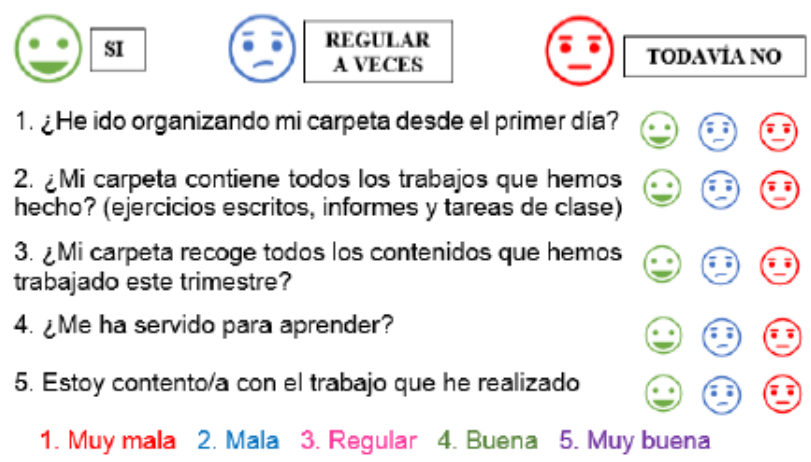

\begin{tabular}{|l|l|l|l|l|l|}
\cline { 2 - 6 } \multicolumn{1}{c|}{} & $\mathbf{1}$ & $\mathbf{2}$ & $\mathbf{3}$ & $\mathbf{4}$ & $\mathbf{5}$ \\
\hline Organización & & & & & \\
\hline Limpieza y caligrafia & & & & & \\
\hline Ortografia & & & & & \\
\hline Calidad del contenido & & & & & \\
\hline Aprendizajes & & & & & \\
\hline
\end{tabular}

Haz una valoración general de tu trabajo y tu aprendizaje y piensa cómo puedes mejorarlo

Figura 1. Ficha de autoevaluación de la carpeta. Fuente: elaboración propia.

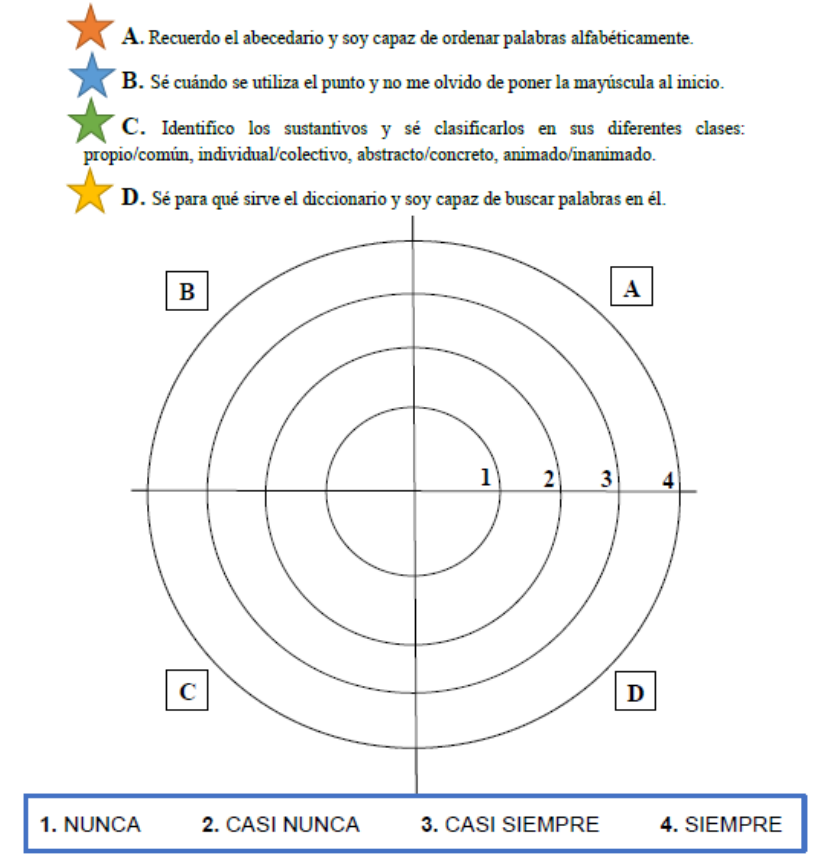

Figura 2. Ejemplo de diana de autoevaluación. Fuente: elaboración propia.

Informes de los talleres: cada grupo rellenará un informe final con información sobre su aprendizaje y la valoración del taller. En otra sesión, se desarrollará una asamblea en la que cada grupo compartirá el trabajo del taller y sus aprendizajes. Tras la asamblea, el docente recogerá los informes y las producciones.

Cuaderno del profesor para recoger hechos significativos. La información puede desembocar en entrevistas no estructuradas individuales o grupales con el alumnado o las familias. Además, se registrará una autoevaluación trimestral, derivada de la heteroevaluación del alumnado, y de la información obtenida de otros instrumentos ¿Cómo pasaremos de la evaluación a la calificación? (Tabla 2).

En la evaluación de los bloques emplearemos fichas con los criterios de evaluación de las unidades del primer trimestre (véase ejemplo de un bloque en el Anexo). Serán 
cumplimentadas por el docente a partir de la información de los ejercicios escritos y mediante la registrada en el cuaderno del profesor. En el caso de que hubiese alguna duda, se consultaría el dossier.

Tabla 2.

Distribución de la calificación trimestral

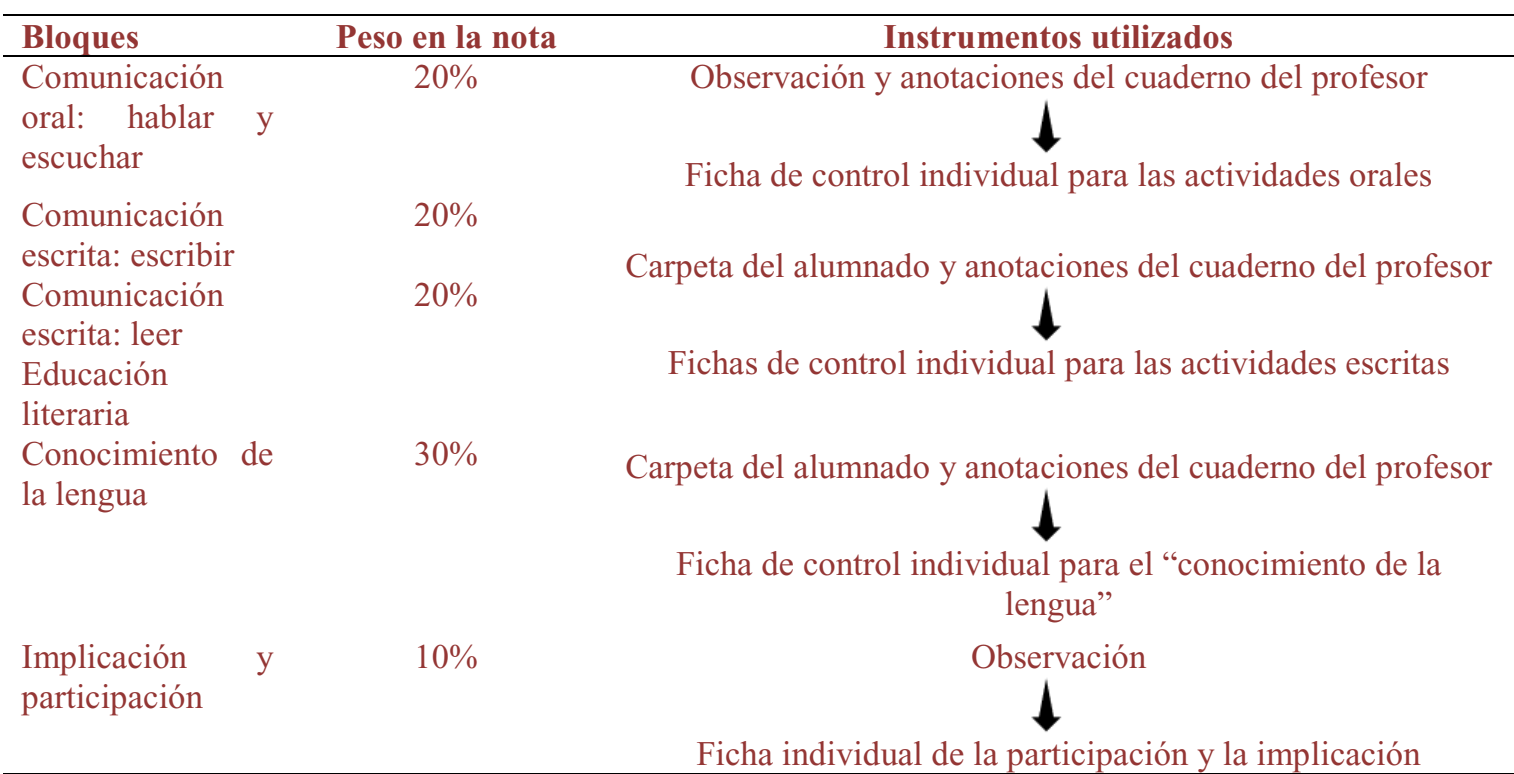

La participación e implicación se valorará mediante la observación, las anotaciones del cuaderno del profesor y la calidad de los productos. La calificación final estará construida por la suma de la nota numérica obtenida en cada tabla, siendo obligatorio alcanzar al menos la mitad de la puntuación en cada bloque.

\section{Evaluación y conclusiones}

El sistema de evaluación formativa fue diseñado para un contexto hipotético durante el Máster de Investigación en Educación (Universidad de Valladolid). No obstante, concluimos que el modelo evaluativo puede promover una mejora en el aprendizaje del alumnado, en el proceso de E-A y en la práctica docente.

\section{Referencias}

Fraile, A., López-Pastor, V., Castejón, F. J. C., \& Romero, R. (2013). La evaluación formativa en docencia universitaria y el rendimiento académico del alumnado. Aula abierta, 41(2), 23-34. 
Gargallo, B., Suárez, J., \& Ferreras, A. (2007). Estrategias de aprendizaje y rendimiento académico en estudiantes universitarios. Revista de Investigación Educativa, 5(2), 421-441.

Hamodi, C., López-Pastor, A., \& López-Pastor, V. (2015). Percepciones de alumnos, egresados y profesores sobre los sistemas de evaluación del aprendizaje. @tic. revista d'innovació educativa, (14), 71-81.

López-Pastor, V. (2012). Evaluación Formativa y Compartida en la Universidad: clarificación de conceptos y propuestas de intervención desde la Red Interuniversitaria de Evaluación Formativa. Psychology, Society \& Educatión, 4(1), 117-130.

López-Pastor, V., \& Pérez-Pueyo, Á. (2017). Buenas prácticas docentes. Evaluación formativa y compartida en educación: experiencias de éxito en todas las etapas educativas. León: Universidad de León. 
Anexo. Ejemplo de ficha de control. Fuente: elaboración propia.

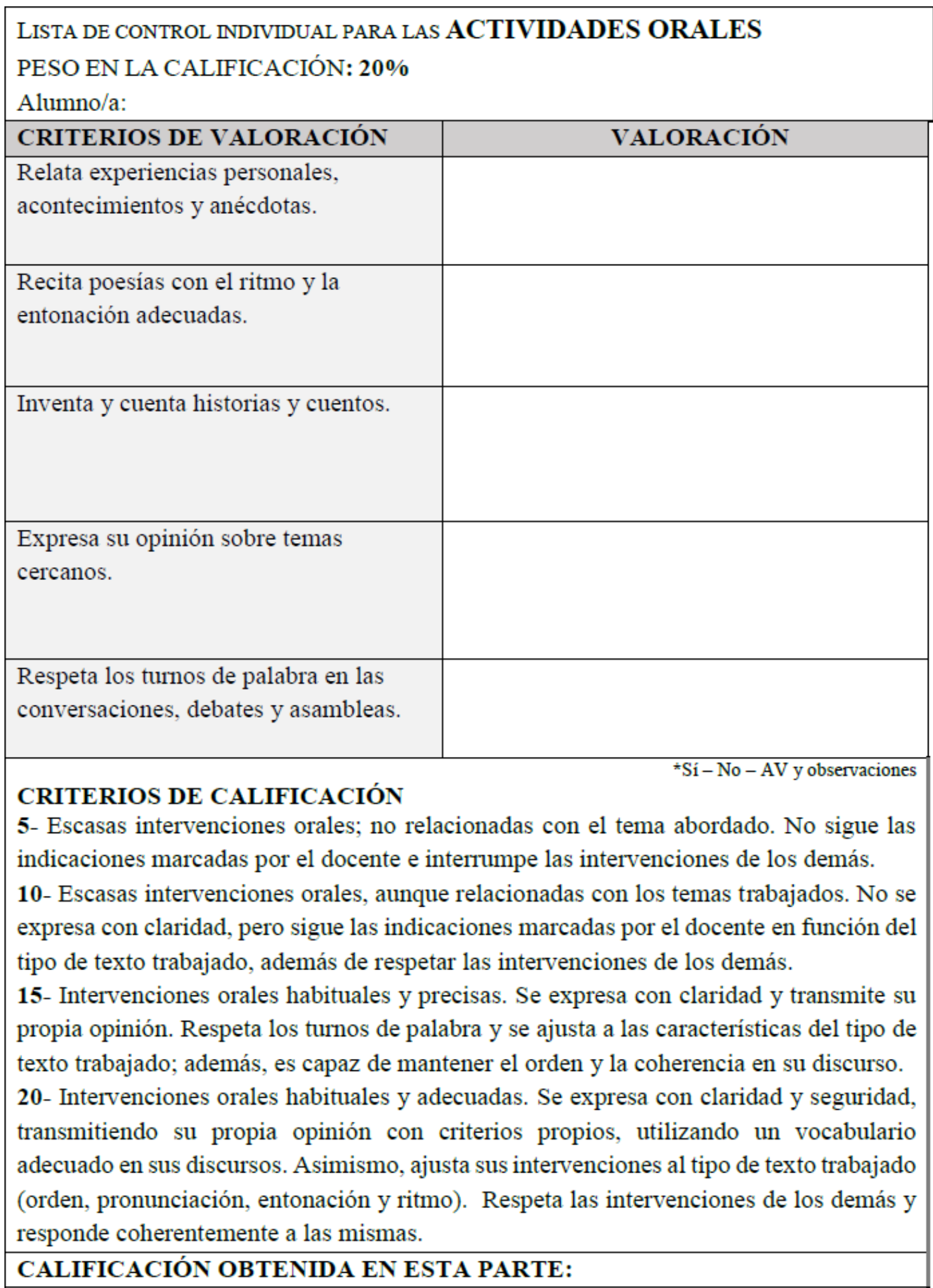

